\title{
Erosividade das chuvas e tempo de recorrência para Lavras, Minas Gerais
}

\author{
Regimeire Freitas Aquino ${ }^{1}$, Marx Leandro Naves Silva ${ }^{2}$, Diego Antonio França de Freitas ${ }^{3}$, Nilton Curi ${ }^{2}$, \\ Carlos Rogério de Mello ${ }^{4}$ Junior Cesar Avanzi $i^{5}$
}

\section{RESUMO}

A erosividade das chuvas é um dos principais agentes causadores da erosão do solo, no sul de Minas Gerais. Por essa razão, objetivou-se, com este trabalho, aplicar cinco distribuições de probabilidades aos valores de erosividades, mensais e anual, e estimar o tempo de recorrência desses valores, com base na melhor distribuição de probabilidades ajustada para a região de Lavras, MG. A série de precipitações estudada foi de 1961 a 2004 e as distribuições de probabilidades utilizadas foram: Gumbel, Gama, Log-normal 2 e 3 parâmetros e Normal, ajustadas pelo método da Máxima Verossimilhança. Verificou-se que as distribuições Gama e Gumbel produziram o menor número de séries não adequadas, registrando-se apenas uma, para o mês de agosto. As demais distribuições produziram maiores números de séries não adequadas, especialmente, a distribuição Log-normal 2 parâmetros. Sendo assim, recomenda-se, para o cálculo de erosividade, que seja feita uma verificação prévia da melhor distribuição para cada período da série estudada, apesar de os dados apontarem um desempenho considerável da distribuição Gumbel.

Palavras-chave: erosão hídrica, distribuição de probabilidade, banco de dados.

\section{ABSTRACT}

\section{Rainfall erosivity and recurrence time for Lavras, Minas Gerais, Brazil}

Rainfall erosivity is a major causative agent of soil erosion in the south region of Minas Gerais state, Brazil. Thus, this study aimed to apply five distributions of probabilities to monthly and annual erosivity and estimate the recurrence time of these values with the best distribution of probability adjusted to the region of Lavras, MG. The precipitation series from 1961 to 2004 was used in the study and the distributions of probabilities were Gumbel, Gamma, Log-normal 2 and 3 parameters and Normal. The distributions Gamma and Gumbel produced the smallest number of non-adequate series, with only one recorded for the month of August. The other distributions produced a larger number of nonadequate series, especially, the Log-normal 2 parameters. Thus, it is recommended that for the calculation of erosivity, a previous assessment of the best distribution for each period of the studied series should be done, despite the data pointing out a considerable performance of the Gumbel distribution.

Key words: water erosion, distribution of probabilities, data base.

Recebido para publicação em 01/06/2012 e aprovado em 04/07/2013.

${ }^{1}$ Engenheira Agrícola, Doutora. Departamento de Agricultura, Instituto Federal de Educação, Ciência e Tecnologia do Sudeste de Minas Gerais, Rua Patronato, s/n, Lindo Vale, 36307-384, São João Del Rei, Minas Gerais, Brasil. regimeire.aquino@ifsudestemg.edu.br

${ }^{2}$ Engenheiro-Agrônomo, Doutor. Departamento de Ciência do Solo, Universidade Federal de Lavras, Campus UFLA, s/n, Caixa Postal 3037, 37200-000, Lavras, Minas Gerais, Brasil.marx@dcs.ufla.br; niltcuri@dcs.ufla.br

${ }^{3}$ Engenheiro-Agrônomo, Doutor. Departamento de CiênciasAgrárias, Universidade Federal de São João del-Rei, Rodovia MG 424, Km 47, Caixa Postal 56, 35701-970, Sete Lagoas, Minas Gerais, Brasil. diego@ufsj.edu.br (autor para correspondência).

${ }^{4}$ Engenheiro Agrícola, Doutor. Departamento de Engenharia, Universidade Federal de Lavras, Campus UFLA, s/n, Caixa Postal 3037, 37200-000, Lavras, Minas Gerais, Brasil. crmello@deg.ufla.br

${ }_{5}^{5}$ Engenheiro Agrícola, Doutor. Embrapa - Centro Nacional de Pesquisa em Pesca, Aquicultura e Sistemas Agrícolas, 77015-012, Palmas, Tocantins, Brasil. junior.avanzi@embrapa.br 


\section{INTRODUÇÃO}

O conhecimento do comportamento e distribuição da erosividade fornece subsídio para determinar períodos críticos predominantes e gerar informações que visem a redução das perdas de solo. Assim, é de grande importância a estimativa das erosividades anuais e mensais, por ser a erosividade o fator mais importante, envolvido na erosão hídrica (Martins et al., 2010).

A determinação dos valores de erosividade permite identificar os períodos do ano nos quais o risco de erosão é mais elevado, o que é importante no planejamento do uso da terra (Mello et al., 2013). No entanto, o tempo de recorrência é um parâmetro da erosividade de elevada importância, definido como o intervalo médio de tempo, em longo prazo, ou o número de anos que separam um evento de dimensão conhecida de outro evento com dimensão igual ou superior, sendo, ao menos uma vez, em qualquer ano, seu valor igualado ou superado (Haan, 1979).

A determinação do tempo de recorrência pode ser conduzida por meio de distribuições de probabilidades, as quais buscam modelar a frequência de ocorrência. Para estudos vinculados à precipitação, e demais grandezas associadas a esta, como a erosividade, as distribuições mais comumente aplicadas são Gumbel, Gama, Log-Normal 2 e 3 parâmetros (Freitas et al., 2001). Dessa forma, os valores anuais e mensais de determinado evento aproximam-se de um padrão definido de distribuição de frequência, quando o número de observações, em cada ano, torna-se o mais amplo possível (Schwab et al., 1966). No caso de tempos de recorrência altos, adota-se o procedimento da distribuição de frequência, estimada por lei de probabilidade teórica, possibilitando um cálculo mais preciso da probabilidade.

Sob condições climáticas extremas, a distribuição de Gumbel tem-se mostrado adequada para descrever esses eventos, principalmente quando se trata de precipitações máximas. Sabendo-se que a erosividade está intimamente relacionada com as intensidades e precipitações máximas, a escolha dos modelos para estimativa da erosividade foi baseada nos estudos relacionados com a precipitação, já existentes.

Estudando o ajuste da distribuição de Gumbel a dados de precipitação pluviométrica máxima diária, coletados no período de 1914 a 1986, na região de Lavras, Gomes et al. (1989) concluíram que os dados de chuvas máximas diárias ajustaram-se à referida distribuição, em períodos de um ano e um mês, exceto para o mês de julho; em períodos de 10 e 15 dias houve ajustamento do modelo somente na estação chuvosa. Back et al. (2011) afirmam que, em estudos sobre distribuições de probabilidades aplicadas a chuvas intensas, a distribuição Gumbel é a que tem produzido melhores ajustes.
Bazzano et al., (2007) analisaram o período de retorno de chuvas, na cidade de Quaraí, RS, e Bazzano et al., (2010) fizeram o mesmo estudo para todo o Rio Grande do Sul, utilizando o modelo de distribuição de Gumbel. Pinto (1995) analisou a relação entre chuvas intensas de diferentes durações e a chuva com duração de 24 horas, para 29 estações pluviométricas, distribuídas no Estado de Minas Gerais, visando a obtenção de fatores de conversão que possibilitem a estimativa de chuvas de curta duração, a partir de informações advindas de pluviômetros. Para tanto, foram testadas as distribuições Gumbel, Log-normal a 2 e 3 parâmetros, empregando períodos de retorno de 2, 5, 10 e 20 anos. O autor concluiu que as distribuiçõos de Gumbel e Log-normal a 2 parâmetros foram as que melhor se ajustaram aos dados observados.

Mello \& Viola (2013), por meio de geoestatística multivariada, promoveram o mapeamento de chuvas intensas, no Estado de Minas Gerais, e observaram maiores intensidades nas regiões Triângulo Mineiro, noroeste e leste de Minas Gerais, sendo esta última a que apresentou os maiores valores de chuvas intensas e, portanto, é a mais crítica em termos de susceptibilidade à erosão hídrica e inundações. Para todas as durações de chuvas intensas estudadas, as regiões norte e nordeste apresentaram as menores intensidades. Aquino et al. (2012) observaram que a região sul de Minas Gerais apresenta elevada erosividade das chuvas, sendo os maiores índices observados nas áreas de maiores altitudes e nos planaltos e serras elevadas.

A distribuição Gama pode ser aplicada em vários estudos, pois é uma função flexível, podendo assumir ou ajustar-se a diferentes tipos de modelos, passando por diversos graus de assimetria. Desta forma, Sampaio et al. (2007) afirmam que a distribuição Gama tem sido bastante utilizada em estudos de predição pluviométrica.

Conforme Moretti \& Mendes (2003), o método da máxima verossimilhança é o mais utilizado para a obtenção de estimadores. Esse método pressupõe que a função de verossimilhança seja conhecida ou pressuposta e atua maximizando a função de densidade de probabilidades, em relação aos efeitos fixos e aos componentes de variância dos efeitos aleatórios do modelo, sempre fornecendo estimativas positivas de componentes de variância (Mello et al., 2008). A interpretação da função de verossimilhança reflete a probabilidade máxima de a amostra ter sido retirada da população da qual foi verdadeiramente retirada.

Este trabalho teve como objetivo aplicar cinco distribuições de probabilidade para valores de erosividade, mensais e anuais, verificando o melhor ajuste e determinando o tempo de recorrência da erosividade da chuva, para Lavras, MG. 


\section{MATERIAL E MÉTODOS}

\section{Estimativa da erosividade}

Foram utilizados dados de precipitação registrados em pluviogramas, correspondentes ao período de 1931 a 2004, coletados na Estação Climatológica Principal de Lavras, MG, e obtidos junto ao Instituto de Meteorologia de Minas Gerais (IMNET-MG).

Para o período de 1961 a 1985, o valor da erosividade $\left(\mathrm{EI}_{30}\right)$ foi obtido por meio do trabalho de Val et al. (1986), o qual utilizou a metodologia proposta por Wischmeier \& Smith (1958) conforme equação (1):

$\mathrm{EC}=0,119+0,0873 \log (\mathrm{I})$

em que EC é a energia cinética da chuva $\left(\mathrm{MJ} \mathrm{ha}^{-1} \mathrm{~mm}^{-1}\right)$ e I é a intensidade da chuva $\left(\mathrm{mm} \mathrm{h}^{-1}\right)$.

$\mathrm{O} \mathrm{EI}_{30}$ foi obtido a partir da multiplicação da energia cinética total pela máxima intensidade ocorrida, por um período de 30 minutos consecutivos $\left(\mathrm{I}_{30}\right)$.

Para o período de 1986 a 2004, foi gerada uma nova equação para a determinação do $\mathrm{EI}_{30}$, conforme equação (2).

$$
\begin{gathered}
E I_{30}=85.672 * R c^{0.6557} \quad \mathrm{r}=0,9786 \\
R c=p^{2} P^{-1},
\end{gathered}
$$

em que:

Rc - coeficiente de chuva (mm);

$\mathrm{p}$ - precipitação média mensal $(\mathrm{mm})$;

$\mathrm{P}$ - precipitação média anual ( $\mathrm{mm})$.

Às frequências observadas das 13 séries históricas de erosividade foram ajustadas as distribuições de probabilidades Gumbel, Gama, Log-normal 3 parâmetros, Lognormal 2 parâmetros e Normal. As estimativas dos parâmetros foram realizadas com base na metodologia da Máxima Verossimilhança, conforme descrito em Clarke (2001), Maranzano \& Krzystofowicz (2004) e Casella \& Berger (1990).

\section{Máxima Verossimilhança}

Segundo Clarke (2001) e Katz et al. (2002), a estimativa dos parâmetros das distribuições de probabilidades, por meio da Máxima Verossimilhança, produz maior precisão das estimativas das frequências observadas, por maximizarem a probabilidade de ocorrência dos valores observados. Isto significa que, dos possíveis valores para um determinado parâmetro, espera-se que o mais adequado seja aquele que maximizar a probabilidade dos valores observados. É em cima desta premissa que a Máxima Verossimilhança é matematicamente definida conforme equação (3):
$\mathrm{L}(\theta ; \mathrm{x} 1 \ldots \mathrm{xn})=\prod_{i=1}^{\mathrm{H}} \mathrm{f}(\mathrm{xi} ; \theta)$

em que $\theta$ é um vetor que representa o conjunto de parâmetros da respectiva função de probabilidades, $x$ representa a variável hidrológica e, f, a distribuição de probabilidades. Esta expressão significa que a verossimilhança de uma distribuição com $\theta$ parâmetros é dada pela soma dos produtos da função. Com o objetivo de linearizar a equação (3), trabalha-se com o logaritmo de L, de acordo com a equação (4):

$$
1(\theta)=\sum_{i=1}^{11} \log f(x i ; \theta)
$$

\section{Distribuição Gumbel}

A Função Densidade de Probabilidade (FDP), da distribuição Gumbel, é dada pela equação (5) (Beijo, 2002):

FDP: $f(x)=b \cdot \exp (-b \cdot(x-u)-\exp (-b \cdot(x-u)))$

em que u e b são parâmetros da distribuição.

A função logaritmo de verossimilhança é dada pela equação (6):

$1(u, b)=-n \cdot \operatorname{Ln}(b)+\sum_{i=1}^{n}\left\lfloor-\left(\frac{x i-u}{b}\right)\right\rfloor-\sum_{i=1}^{n} \exp \left\lfloor-\left(\frac{x i-u}{b}\right)\right.$.

A partir da primeira derivada parcial da equação (6), em relação a u e b, é possível obter o ponto de máximo desta função e seus melhores parâmetros de ajuste, conforme equações (7) e (8):

$$
\begin{aligned}
& u=-b \cdot \operatorname{Ln}\left\lfloor\frac{1}{n} \cdot \sum_{i=1}^{n} \exp \left(\frac{-x i}{b}\right)_{-}\right. \\
& b=\frac{1}{n} \sum_{i=1}^{n} x i-\frac{\sum_{i=1}^{n} x i \cdot \exp \left(\frac{-x i}{b}\right)}{\sum_{i=1}^{n} \exp \left(\frac{-x i}{b}\right)}
\end{aligned}
$$

A equação (8) não apresenta solução explícita para b, havendo necessidade de aproximação numérica, a qual foi realizada com base no método de Newton-Raphson, para posterior obtenção direta de u na equação (7).

\section{Distribuição Gama}

A FDP da distribuição Gama incompleta (2 parâmetros) é dada pela equação (9) (Haan, 1979):

FDP: $f(x)=\frac{1}{\beta^{\alpha} \cdot \Gamma(\alpha)} \cdot x^{\alpha-1} \cdot \exp (-x / \beta$, 
A função de log-verossimilhança é dada pela equação (10):

$1(\alpha, \beta)=-n \cdot \alpha \cdot \operatorname{Ln}(\beta)-n \cdot \operatorname{Ln}[\Gamma(\alpha)]+(\alpha-1) \cdot \operatorname{Ln}\left[\sum \operatorname{Ln}(x i)\right]$ $-\frac{\sum x}{\beta}$

As derivadas parciais da equação (10), em relação a $\alpha$ e $\beta$, igualadas a zero, produzem as equações (11) e (12):

$\operatorname{Ln}(\alpha)-\psi(\alpha)=\operatorname{Ln}\left(\bar{X} \frac{}{X_{G}}\right)$

$\beta=\frac{\bar{x}}{\alpha}$

em que $\bar{X}$ e $\bar{X}_{\mathrm{G}}$ são, respectivamente, as médias aritmética e geométrica dos dados, e $\psi(\alpha)$ é a função digama de $\alpha$, aproximada pela equação (13):

$\psi(\alpha) \cong \operatorname{Ln}(\alpha)-\frac{1}{2 \cdot \alpha}-\frac{1}{12 \cdot \alpha^{2}}+\frac{1}{120 \cdot \alpha^{4}}-\frac{1}{252 \cdot \alpha^{6}}+$ $+\frac{1}{240 \cdot \alpha^{8}}-$

Para solução da equação (11) foi desenvolvido um algoritmo computacional para estimativa do parâmetro $\alpha$, com base também no método de Newton-Raphson, uma vez que o mesmo está implícito nesta equação.

\section{Distribuição Log-Normal 3 parâmetros}

A Função Densidade de Probabilidades da distribuição Log-normal 3 parâmetros é dada pela equação (14) (Clarke, 2001):

FDP: $f(x)=\frac{1}{(x-a) \cdot \sigma \cdot \sqrt{2 \cdot \pi}} \cdot \exp \left(\frac{-1}{2 \cdot \sigma^{2}} \cdot(\log (x-a)-\mu)^{2}\right.$

em que $\mu, \sigma$ e a são parâmetros. Adotando-se $x^{*}=x-a$, a estimativa de $\mu$ e $\sigma$, por Máxima Verossimilhança, é dada pelas equações (15) e (16):

$\mu=\frac{\sum_{i=1}^{n}\left(\log x_{i}\right)}{n}$

$\sigma=\sqrt{\frac{\sum_{i=1}^{n}\left(\log \left(x_{i}-\mu\right)^{2}\right)}{n}}$

O parâmetro a é testado por vários valores, avaliandose a função logaritmo de verossimilhança, em que o valor que maximizar esta função é adotado como parâmetro da distribuição de probabilidades. Para obtenção desses parâmetros, também foi desenvolvido um algoritmo para teste dos valores de a e posterior determinação do logverossimilhança, pela equação (17): $1(\mu, \sigma, a)=-n \cdot \log \sigma-\frac{n \cdot \log (2 \pi)}{2}-\sum_{i=1}^{n} \log (x * i)-$

$-\frac{1}{2 \cdot \sigma^{2}} \cdot \sum_{i=1}^{n}\left(\log x_{i}-\mu\right)^{2}$

\section{Distribuição Log-Normal 2 parâmetros}

A FDP para a distribuição Log-normal 2 parâmetros é dada pela equação (18):

FDP: $f(x)=\frac{1}{x \cdot \sigma^{*} \cdot \sqrt{2 \cdot \pi}} \cdot \exp \left(\frac{-1}{2 \cdot \sigma^{2}} \cdot\left(\log (x)-\mu^{*}\right)^{2}\right)$

em que $\sigma^{*}$ e $\mu^{*}$ são parâmetros desta distribuição, cuja estimativa por Máxima Verossimilhança é dada pelas equações (19) e (20):

$\mu^{*}=\frac{\sum_{i=1}^{n}(\log (x i))}{n}$

$\sigma^{*}=\sqrt{\frac{\sum_{i=1}^{n}(\log (x i)-\mu)^{2}}{n}}$

A FDP da Distribuição Normal é dada pela equação (21):

FDP: $f(x)=\frac{1}{\sigma \cdot \sqrt{2 \pi}} \cdot \exp \left(-0,5 \cdot\left(\frac{x-\mu}{\sigma}\right)^{2}\right)$

Os parâmetros $\mu$ e $\sigma$ são estimados pela Máxima Verossimilhança como, respectivamente, a média e o desvio padrão dos dados (Clarke, 2001).

\section{Adequabilidade das distribuições}

Para fins de análise da precisão, ao ajuste das distribuições de frequência, além da adequabilidade propriamente dita, aplicou-se o teste de $\chi^{2}$ (Qui-Quadrado). Segundo Catalunha et al. (2002), esse teste é um excelente indicador do ajuste das distribuições de probabilidades, por refletir o quadrado médio do erro, conforme equação (22):

$\chi_{\text {cal }}^{2}=\frac{\sum_{i=1}^{n}\left(f_{\text {obsi- }} f_{\text {teori }}\right)^{2}}{f_{\text {tenri }}}$

em que $\mathrm{f}_{\text {obsi }}$ e $\mathrm{f}_{\text {teori }}$ são as frequências observada e teórica, na respectiva classe de frequência $i, e, n$, o número de classes. A distribuição é adequada se $\chi_{\text {cal }}^{2}$ for menor que $\chi_{\text {tabela }}^{2}$, a mais precisa, obtida a partir do menor valor de $\chi^{2}$. O valor de $\chi_{\text {tabela }}^{2}$ foi obtido, considerando-se como graus de liberdade um valor intermediário entre (n-1) e (n$\mathrm{p}_{\mathrm{p}}-1$ ), em que $\mathrm{p}_{\mathrm{p}}$ é o número de parâmetros do modelo de probabilidades, pela incerteza a respeito do valor exato deste, conforme descrito por Ferreira (2005). Assim foi adotado o valor (n-2) para graus de liberdade para todos 
os modelos de probabilidade deste trabalho. Uma vez avaliada a distribuição de maior precisão para as séries estudadas, foram calculadas as erosividades associadas a diferentes probabilidades.

\section{Tempo de recorrência}

O tempo de recorrência (TR) expressa a probabilidade de um determinado evento climatológico (chuva, vazão, etc) ser igualado ou superado, pelo menos uma vez, em qualquer ano, até o seu valor máximo, em anos (Haan, 1979). Assim, o TR pode ser estimado segundo a equação (23):

$$
\mathrm{TR}=\frac{1}{\mathrm{~F}\left(\mathrm{X}_{\mathrm{TR}} \geq \mathrm{x}\right)} \cong \frac{1}{\operatorname{Prob}\left(\mathrm{X}_{\mathrm{TR}} \geq \mathrm{x}\right)}(23),
$$

em que TR é obtido em anos e Prob $\left(\mathrm{X}_{\mathrm{TR}} \geq \mathrm{x}\right)$ é a probabilidade de excedência, ou seja, de o valor de $\mathrm{X}$ ser igualado ou superado.

\section{RESULTADOS E DISCUSSÃO}

Os valores de $\chi^{2}$ calculados para cada uma das distribuições de probabilidades ajustadas às séries históricas, os valores de $\chi^{2}$ tabelado e a respectiva adequação das distribuições estão apresentados na Tabela 1. Observase que as distribuições Gama e Gumbel foram as que produziram o menor número de séries não adequadas, registrando-se apenas uma, para o mês de agosto. As demais distribuições produziram maiores quantidades de séries não adequadas, especialmente a distribuição log-normal 2 parâmetros, que produziu 6, dentro das 13 séries. A distribuição log-normal 3 parâmetros produziu não adequabilidade para 3 séries, sendo que este comportamento foi produzido pela não convergência do parâmetro a da dis- tribuição, ou seja, não foi possível encontrar um ponto de máximo da função log-verossimilhança, pelo fato de a função apresentar mais de um ponto de máximo (equação 18).

No entanto, ao se analisarem os valores de $\chi^{2}$ para cada série histórica, verifica-se que houve variações da melhor distribuição, pelo que se observou que a distribuição Gumbel foi melhor para as séries de abril, maio e setembro, log-normal 2 parâmetros para janeiro, julho e agosto, Gama para junho e novembro e a distribuição log-normal 3 parâmetros foi a que produziu a maior quantidade de séries com o menor valor de $\chi^{2}$, tendo sido a de melhor ajuste para fevereiro, março, outubro, dezembro e anual. A distribuição Normal mostrou-se não adequada para apenas três meses, no entanto, os valores de $\chi^{2}$ são consideravelmente elevados em relação aos das demais distribuições, não sendo recomendada para estimativas da erosividade.

Observa-se ainda que as distribuições produziram valores de $\chi^{2}$ menores para séries históricas pertencentes ao período chuvoso, ou seja, entre outubro e março, e as séries anuais, havendo melhor ajuste das distribuições. Nesses casos, apenas as distribuições Normal e log-normal 2 parâmetros produziram valores mais elevados, sendo esta última, em alguns casos, não adequada. Assim, nesse período, pode-se concluir que qualquer uma das demais distribuições pode produzir bons resultados, especialmente a log-normal 3 parâmetros. Ribeiro et al. (2007), analisando a precipitação no período chuvoso em Barbacena (MG), também encontraram resultados semelhantes, sendo a distribuição log-normal 3 parâmetros a que apresentou os menores valores do Qui-Quadrado, para o período mensal.

Tabela 1. Valores de Qui-Quadradro $\left(\mathrm{c}^{2}\right)$ produzidos pelas respectivas distribuições de probabilidades ajustadas às séries históricas, mensais e anuais, de erosividade, para a região de Lavras (MG), e respectiva adequação

\begin{tabular}{|c|c|c|c|c|c|c|c|c|c|c|c|}
\hline \multirow[t]{2}{*}{ Série } & \multirow[t]{2}{*}{$\chi^{2}$} & \multicolumn{2}{|c|}{ Gumbel } & \multicolumn{2}{|c|}{$\begin{array}{c}\text { Log normal } 2 \\
\text { parâmetros }\end{array}$} & \multicolumn{2}{|c|}{$\begin{array}{c}\text { Log normal } 3 \\
\text { parâmetros }\end{array}$} & \multicolumn{2}{|c|}{ Normal } & \multicolumn{2}{|c|}{ Gama } \\
\hline & & Valores & AD & Valores & AD & Valores & AD & Valores & AD & Valores & AD \\
\hline Jan. & 7,81 & 1,861 & A & 1,134 & A & $0,979 *$ & A & 7,388 & A & 1,164 & A \\
\hline Fev. & 7,81 & 4,002 & $\mathrm{~A}$ & $2,536^{*}$ & A & 9,166 & NA & 2,632 & A & 4,771 & A \\
\hline Mar. & 5,99 & 0,264 & $\mathrm{~A}$ & 0,251 & A & 7,269 & NA & 5,739 & A & 0,383 & A \\
\hline Abr. & 5,99 & $0,594 *$ & A & 2,851 & A & 10,679 & NA & 3,179 & A & 0,993 & A \\
\hline Mai. & 5,99 & $3,303 *$ & A & $\mathrm{SC}$ & NA & 17,518 & $\mathrm{NA}$ & 4,521 & A & 3,800 & A \\
\hline Jun. & 3,81 & 0,368 & A & $\mathrm{SC}$ & NA & 2,653 & A & 6,244 & NA & $0,314 *$ & A \\
\hline Jul. & 3,81 & 2,527 & A & 2,025 & A & $1,593 *$ & A & 6,716 & NA & 2,819 & A \\
\hline Ago. & 5,99 & 7,101 & NA & $\mathrm{SC}$ & NA & $3,093 *$ & A & 9,831 & NA & 11,525 & NA \\
\hline Set. & 9,49 & $1,783^{*}$ & A & 5,704 & A & 20,402 & NA & 3,150 & A & 6,576 & A \\
\hline Out. & 9,49 & 0,835 & A & $0,546^{*}$ & A & 13,977 & NA & 0,982 & A & 1,871 & A \\
\hline Nov. & 7,81 & 0,713 & A & 0,481 & A & 1,072 & A & 0,883 & A & $0,126^{*}$ & A \\
\hline Dez. & 5,99 & 1,091 & A & $1,021 *$ & $\mathrm{~A}$ & 4,571 & A & 2,997 & $\mathrm{~A}$ & 2,804 & $\mathrm{~A}$ \\
\hline Anual & 5,99 & 1,623 & A & $1,168^{*}$ & $\mathrm{~A}$ & 1,202 & A & 1,705 & $\mathrm{~A}$ & 4,797 & $\mathrm{~A}$ \\
\hline
\end{tabular}

(*) distribuições sugeridas para cálculo da erosividade associada aos tempos de recorrência. $\mathrm{AD}=$ ajuste a distribuição; $\mathrm{A}=$ adequação; $\mathrm{NA}=$ não adequação; $\mathrm{SC}=$ sem convergência dos parâmetros no respectivo algoritmo. 
As séries históricas do período seco, entre abril e setembro, foram as que apresentaram maiores dificuldades de ajuste das distribuições. Isto acontece por causa da elevada variabilidade dos dados da série, podendo variar de valores próximos a zero até acima de 100, aumentando seu coeficiente de variação. Além disto, a grande quantidade de zeros dentro da série faz com que as frequências estimadas fiquem distantes das observadas, especialmente para baixos valores de erosividade, contribuindo para sua não adequação. É possível observar elevada concentração de séries não adequadas neste período, especialmente para o mês de agosto, para o qual apenas a distribuição log-normal 2 parâmetros foi adequada. Sendo assim, recomenda-se que, para cálculos da erosividade neste período, seja feita uma verificação prévia da melhor distribuição, apesar de os dados da Tabela 1 apontarem um desempenho considerável da distribuição Gumbel neste período.

Conforme trabalhos de Araújo et al. (2001), os períodos secos não se ajustaram à distribuição Gama. Estes mesmos autores identificaram, pela distribuição normal, ajuste dos meses de abril a novembro e para o total anual; entretanto, a aderência das frequências estimadas para as precipitações mensais à curva de Gauss foi inferior à aderência à curva da distribuição Gama, razão pela qual estes autores recomendam o uso da distribuição Gama, em estudos visando à estimativa da precipitação provável.

Os valores de erosividades mensais e anuais estimados pela melhor distribuição de probabilidade ajustada para a região de Lavras (MG) estão apresentados na Tabela 2. Pode-se observar que os maiores valores de erosividade ocorrem de outubro a março e, no período de abril a setembro, são consideravelmente menores. Essa distribuição temporal é característica da região, com verão chuvoso e inverno seco, sendo que vários pesquisadores, em diversas regiões do país, têm encontrado situ- ação semelhante (Dias \& Silva, 2003; Silva et al., 2009; Bertoni \& Lombardi Neto, 2010; Silva et al., 2010; Aquino et al., 2012; Aquino et al., 2013).

De acordo com Foster et al. (1981), erosividades anuais entre 7.357 e $9.810 \mathrm{MJ} \mathrm{mm} \mathrm{ha}^{-1} \mathrm{~h}^{-1}$ ano $^{-1}$ são consideradas "altas" e, acima de $9.810 \mathrm{MJ} \mathrm{mm} \mathrm{ha}^{-1} \mathrm{~h}^{-1} \mathrm{ano}^{-1}$, classificadas como "muito altas". Assim, a erosividade média de Lavras pode ser enquadrada na classe "alta"; contudo, para TR acima de 20 anos, esta passa a ser considerada como "muita alta". Isto significa que existe uma probabilidade de $5 \%$ de a erosividade ser considerada como crítica, na região de Lavras, num ano qualquer, o que é significativo, em se tratando de riscos à erosão e movimentação de massas de solo. Para os meses de outubro a março, todas as erosividades estimadas mostraram-se maiores que $500 \mathrm{MJ} \mathrm{mm} \mathrm{ha}^{-1} \mathrm{~h}^{-1}$ mês $^{-1}$ e, segundo Oliveira et al. (2009), erosividades mensais acima deste valor podem ser consideradas como críticas. Apenas no mês de junho, não houve ocorrência de erosividade crítica.

Para a região de Lavras, no período de plantio e estabelecimento das culturas, entre outubro e dezembro, existe risco potencial de erosão, pois as chuvas apresentam erosividade elevada e o solo encontra-se desprovido de vegetação e, muitas vezes, desagregado, em razão do preparo para o plantio e, consequentemente, pouco protegido (Castro et al., 2011). No início do seu desenvolvimento, as plantas não apresentam cobertura do solo suficiente para dissipar a elevada energia cinética das gotas de chuva em queda livre, o que aumenta as perdas de solo (Cardoso et al., 2012). Desta forma, para o dimensionamento de obras hidráulicas, tanto urbanas, como rurais, é necessário o conhecimento do tempo de recorrência das chuvas e a erosividade, de modo que a estrutura planejada possa resistir adequadamente. No caso de obras rurais, esse tipo de conhecimento é ne-

Tabela 2. Valores de erosividades, mensais e anuais, estimados pela melhor distribuição de probabilidades ajustada para a região de Lavras, MG

\begin{tabular}{|c|c|c|c|c|c|c|c|c|c|c|c|c|c|}
\hline \multirow{2}{*}{$\begin{array}{l}\text { TR } \\
\text { Anos }\end{array}$} & Jan. & Fev. & Mar. & Abr. & Mai. & Jun. & Jul. & Ago. & Set. & Out. & Nov. & Dez. & Anual \\
\hline & \multicolumn{13}{|c|}{$\left(\mathrm{MJ} \mathrm{mm} \mathrm{ha}^{-1} \mathrm{~h}^{-1}\right)$} \\
\hline 5 & 2190 & 1425 & 1031 & 384 & 274 & 162 & 34 & 50 & 292 & 674 & 1180 & 2052 & 8071 \\
\hline 10 & 2989 & 1686 & 1376 & 510 & 366 & 219 & 90 & 135 & 383 & 834 & 1460 & 2525 & 8996 \\
\hline 20 & 3868 & 1912 & 1734 & 631 & 455 & 275 & 203 & 309 & 471 & 984 & 1721 & 2976 & 9850 \\
\hline 30 & 4424 & 2033 & 1951 & 701 & 505 & 308 & 309 & 476 & 521 & 1068 & 1872 & 3234 & 10328 \\
\hline 40 & 4833 & 2114 & 2107 & 750 & 541 & 329 & 408 & 632 & 557 & 1126 & 1970 & 3413 & 10656 \\
\hline 50 & 5169 & 2176 & 2233 & 788 & 569 & 346 & 503 & 784 & 584 & 1171 & 2049 & 3555 & 10912 \\
\hline 60 & 5412 & 2218 & 2323 & 819 & 591 & 359 & 581 & 909 & 607 & 1203 & 2105 & 3654 & 11092 \\
\hline 70 & 5706 & 2268 & 2432 & 845 & 610 & 374 & 687 & 1077 & 625 & 1241 & 2172 & 3772 & 11303 \\
\hline 80 & 5953 & 2308 & 2522 & 868 & 627 & 386 & 784 & 1235 & 642 & 1271 & 2225 & 3868 & 11474 \\
\hline 90 & 6103 & 2331 & 2575 & 888 & 641 & 393 & 847 & 1337 & 656 & 1289 & 2255 & 3925 & 11576 \\
\hline 100 & 6278 & 2358 & 2638 & 906 & 654 & 400 & 926 & 1464 & 669 & 1310 & 2288 & 3991 & 11693 \\
\hline
\end{tabular}

$\mathrm{TR}=$ tempo de recorrência

Rev. Ceres, Viçosa, v. 61, n.1, p. 009-016, jan/fev, 2014 
cessário para o planejamento de sistemas de terraceamento agrícola, drenagem em estradas e implantação de barragens para atenuação de cheias, dentre outros (Mesquita et al., 2009).

O período de recorrência utilizado para o terraceamento agrícola normalmente aproxima-se de dez anos (Mesquita et al., 2009), porém, pode-se, eventualmente, planejar essa prática com uma margem de segurança maior, visando a solucionar problemas específicos, considerados os de ordem econômica e ecológica, e os possíveis danos maiores presumíveis, adotando-se períodos de recorrência mais convenientes, mesmo que superestimem a dimensão normal dos canais dos terraços, como de 20 a 50 anos (Moreti et al., 2003).

\section{CONCLUSÕES}

As distribuições Gama e Gumbel apresentaram apenas uma série não adequada, no mês de agosto.

As distribuições Log-normal 3 parâmetros, Log-normal 2 parâmetros e Normal apresentaram maiores quantidades de séries não adequadas, especialmente, a distribuição Log-normal 2 parâmetros.

Para as erosividades mensais do período chuvoso, a distribuição log-normal 3 parâmetros apresentou maior precisão.

As erosividades anuais, para todos os tempos de recorrência, foram consideradas muito altas e os valores das erosividades mensais, para os meses de outubro a março, também podem ser considerados críticos. Apenas o mês de junho não apresentou erosividade considerada crítica, para nenhum tempo de recorrência.

\section{REFERÊNCIAS}

Aquino RF, Silva MLN, Freitas DAF, Curi N \& Avanzi JC (2013) Soil losses from Typic Cambisols and Red Latosol within three different erosive rainfall patterns. Revista Brasileira de Ciência do Solo, 37:213-220.

Aquino RF, Silva MLN, Freitas DAF, Curi N, Mello CR \& Avanzi JC (2012) Spatial variability of the rainfall erosivity in Southern region of Minas Gerais State, Brazil. Ciência e Agrotecnologia, 36:533-542.

Araújo WF, Andrade Júnior AS, Medeiros RD \& Sampaio RA (2001) Precipitação pluviométrica mensal provável em Boa Vista, Estado de Roraima, Brasil. Revista Brasileira de Engenharia Agrícola e Ambiental, 5:563-567.

Back AJ, Henn A \& Oliveira JLR (2011) Heavy rainfall equations for Santa Catarina, Brazil. Revista Brasileira de Ciência do Solo, 35:2127-2134.

Bazzano MG, Eltz FLF \& Cassol EA (2007) Erosividade, coeficiente de chuva, padrões e período de retorno das chuvas de Quarai, RS. Revista Brasileira de Ciência do Solo, 31:1205-1217.

Bazzano MG, Eltz FLF \& Cassol EA (2010) Erosividade e características hidrológicas das chuvas de Rio Grande (RS). Revista Brasileira de Ciência do Solo, 34:235-244.
Beijo LA (2002) Distribuição de Gumbel: estudo de métodos de estimação dos parâmetros e ajuste aos dados de precipitação máxima de Lavras, MG. Dissertação de Mestrado. Universidade Federal de Lavras, Lavras. 91p.

Bertoni J \& Lombardi Neto F (2010) Conservação do solo. Piracicaba, Ícone. 355p.

Cardoso DP, Silva MLN, Carvalho GJ, Freitas DAF \& Avanzi JC (2012) Plantas de cobertura no controle das perdas de solo, água e nutrientes por erosão hídrica. Revista Brasileira de Engenharia Agrícola e Ambiental, 6:632-638.

Casella G \& Berger RL (1990) Statistical Inference. Belmont, Duxbury Press. 650p.

Castro NAE, Silva MLN, Freitas DAF, Carvaho GJ, Marques RM \& Gontijo GF (2011) Plantas de cobertura no controle da erosão hídrica sob chuvas naturais. Bioscience Journal, 27:775-785.

Catalunha MJ, Sediyama GC, Leal BG, Soares CPB \& Ribeiro A (2002) Aplicação de cinco funções densidade de probabilidade a séries de precipitação pluvial no estado de Minas Gerais. Revista Brasileira de Agrometereologia, 10:153-162.

Clarke R (2001) Hidrologia estatísica. In: Tucci CEM (Ed.) Hidrologia: ciência e aplicação. Porto Alegre, Associação Brasileira de Recursos Hídricos. p.659-702.

Dias AS \& Silva JCR (2003) A erosividade das chuvas em Fortaleza (CE). I - Distribuição, probabilidade de ocorrência e período de retorno $-1^{\mathrm{a}}$ Aproximação. Revista Brasileira de Ciência do Solo, 27:335-345.

Ferreira DF (2005) Estatística Básica. Lavras, UFLA. 664p.

Foster GR, McCool DK, Renard KG \& Moldenhauer WC (1981) Conversion of the universal soil loss equation to SI metric units. Journal of Soil and Water Conservation, 36:355-359.

Freitas AJ, Silva DD, Pruski FF, Pinto FA, Pereira SB, Gomes Filho RR, Teixeira AF, Baena LGN, Mello LTA \& Novaes LF (2001) Equações de chuvas intensas no Estado de Minas Gerais. Belo Horizonte, Copasa. 65p.

Gomes FG, Aquino LH \& Oliveira MS (1989) Estudo da distribuição e frequência de precipitação pluviométrica máxima em períodos de dez e quinze dias, um mês e um ano, em Lavras (MG) pela distribuição Gumbel. Ciência e Prática, 13:177-184.

Haan CT (1979) Statistical methods in hydrology. 2a ed. Ames, The Iowa State University Press. 377p.

Katz RW, Parlange MB \& Naveau P (2002) Statistical of extremes in hydrology. Advances in Water Resources, 25:1287-1304.

Maranzano CJ \& Krzysztofowics R (2004) Identification of likelihood and prior dependence structures for hydrologic uncertainty processor. Journal of Hydrology, 290:1-21.

Martins SG, Avanzi JC, Silva MLN, Curi N, Norton LD \& Fonseca S (2010) Rainfall erosivity and rainfall return period in the Experimental Watershed of Aracruz, in the Coastal Plain of Espirito Santo. Revista Brasileira de Ciência do Solo, 34:999-1004.

Mello CR \& Viola MR (2013) Mapeamento de chuvas intensas no estado de Minas Gerais. Revista Brasileira de Ciência do Solo, 37:37-44.

Mello CR, Viola MR, Beskow S \& Norton LD (2013) Multivariate models for annual rainfall erosivity in Brazil. Geoderma, 202:88102 .

Mello CR, Viola MR, Mello JM \& Silva AM (2008) Continuidade espacial de chuvas intensas no Estado de Minas Gerais. Ciência e Agrotecnologia, 32:532-539.

Mesquita WO, Griebeler PN \& Oliveira LFC (2009) Precipitações máximas diárias esperadas para as regiões central e sudeste de Goiás. Pesquisa Agropecuária Tropical, 39:73-81. 
Moreti D, Carvalho MP, Mannigel AR \& Medeiros LR (2003) Importantes características de chuva para a conservação do solo e da água no município de São Manuel (SP). Revista Brasileira de Ciência do Solo, 27:713-725.

Moretti AR \& Mendes BVM (2003) Sobre a precisão das estimativas de máxima verossimilhança nas distribuições bivariadas de valores extremos. Pesquisa Operacional, 23:301-324.

Oliveira FP, Silva MLN, Curi N, Silva MA \& Mello CR (2009) Potencial erosivo da chuva no Vale do Rio Doce, região CentroLeste do estado de Minas Gerais - primeira aproximação. Ciência e Agrotecnologia, 33:1569-1577.

Pinto FRL (1995) Chuvas intensas no estado de Minas Gerais: análises e modelos. Tese de Doutorado. Universidade Federal de Viçosa, Viçosa. 87p.

Ribeiro BT, Avanzi JC, Mello CR, Lima JM \& Silva MLN (2007) Comparação de distribuição de probabilidade e estimativa da precipitação provável para região de Barbacena, MG. Ciência e Agrotecnologia, 31:1297-1302.
Sampaio SC, Queiroz MMF, Frigo EP, Longo AJ \& Suszek M (2007) Estimativa e distribuição de precipitações decendiais para o estado do Paraná. Irriga, 1:38-53.

Schwab GO, Frevert RK, Dminster TW \& Barnes KK (1966) Soil and Water Conservation Engineering. 2 a ed. New York, John Wiley. 683p.

Silva AM, Silva MLN, Curi N, Avanzi JC \& Ferreira MM (2009) Erosividade da chuva e erodibilidade de Cambissolo e Latossolo na região de Lavras, Sul de Minas Gerais. Revista Brasileira de Ciência do Solo, 33:1811-1820.

Silva MA, Silva MLN, Curi N, Santos GS, Marques JJM, Menezes MD \& Leite FP (2010) Espacialização da erosividade da chuva no Vale do rio doce, região Centro-Leste de Minas Gerais. Revista Brasileira de Ciência do Solo, 34:1029-1039.

Val LA, Bahia VG, Freire JC \& Dias Júnior MS (1986) Erosividade das chuvas em Lavras, MG. Ciência e Prática, 10:199-209.

Wischmeier WH \& Smith DD (1958) Rainfall energy and its relationship to soil loss. Transaction America Society of Civil Engineering, 39:285-291. 